\title{
Pathophysiology of SARS-CoV-2: the Mount Sinai COVID-19 autopsy experience
}

\author{
Clare Bryce $^{1} \cdot$ Zachary Grimes $^{1} \cdot$ Elisabet Pujadas ${ }^{1} \cdot$ Sadhna Ahuja $^{1} \cdot$ Mary Beth Beasley ${ }^{1} \cdot$ Randy Albrecht $^{1}$. \\ Tahyna Hernandez ${ }^{1} \cdot$ Aryeh Stock $\mathbb{D}^{1} \cdot$ Zhen Zhao $^{1} \cdot$ Mohamed Rizwan AlRasheed ${ }^{1} \cdot$ Joyce Chen ${ }^{1} \cdot$ Li Li $^{1}$. \\ Diane Wang ${ }^{1}$ - Adriana Corben ${ }^{1}$ - G. Kenneth Haines III $^{1}$ - William H. Westra $\mathbb{D}^{1}$ - Melissa Umphlett ${ }^{1}$. \\ Ronald E. Gordon ${ }^{1} \cdot$ Jason Reidy $^{1} \cdot$ Bruce Petersen $^{1}{ }^{1} \cdot$ Fadi Salem $^{1} \cdot$ Maria Isabel Fiel $^{1} \cdot$ Siraj M. El Jamal ${ }^{1}$. \\ Nadejda M. Tsankova ${ }^{1} \cdot$ Jane Houldsworth ${ }^{1} \cdot$ Zarmeen Mussa $^{1} \cdot$ Brandon Veremis $^{1} \cdot$ Emilia Sordillo $\mathbb{D}^{1}$.

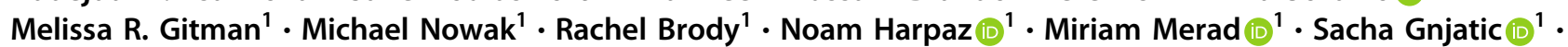 \\ Wen-Chun Liu ${ }^{1} \cdot$ Michael Schotsaert ${ }^{1} \cdot$ Lisa Miorin ${ }^{1} \cdot$ Teresa A. Aydillo Gomez $\mathbb{D}^{1} \cdot$ Irene Ramos-Lopez $^{1}$. \\ Adolfo Garcia-Sastre $^{1} \cdot$ Ryan Donnelly $^{1} \cdot$ Patricia Seigler $^{1} \cdot$ Calvin Keys $^{1} \cdot$ Jennifer Cameron $^{1} \cdot$ Isaiah Moultrie $^{1}$. \\ Kae-Lynn Washington ${ }^{1} \cdot$ Jacquelyn Treatman ${ }^{1} \cdot$ Robert Sebra $^{1} \cdot$ Jeffrey Jhang ${ }^{1} \cdot$ Adolfo Firpo $^{1} \cdot$ John Lednicky $^{2}$. \\ Alberto Paniz-Mondolfi ${ }^{1} \cdot$ Carlos Cordon-Cardo $\mathbb{B}^{1} \cdot$ Mary E. Fowkes ${ }^{1}$
}

Received: 15 July 2020 / Revised: 1 March 2021 / Accepted: 2 March 2021 / Published online: 1 April 2021

(c) The Author(s), under exclusive licence to United States \& Canadian Academy of Pathology 2021

\begin{abstract}
Severe acute respiratory syndrome coronavirus 2 (SARS-CoV-2) and its associated clinical syndrome COVID-19 are causing overwhelming morbidity and mortality around the globe and disproportionately affected New York City between March and May 2020. Here, we report on the first 100 COVID-19-positive autopsies performed at the Mount Sinai Hospital in New York City. Autopsies revealed large pulmonary emboli in six cases. Diffuse alveolar damage was present in over $90 \%$ of cases. We also report microthrombi in multiple organ systems including the brain, as well as hemophagocytosis. We additionally provide electron microscopic evidence of the presence of the virus in our samples. Laboratory results of our COVID-19 cohort disclose elevated inflammatory markers, abnormal coagulation values, and elevated cytokines IL-6, IL-8, and TNF $\alpha$. Our autopsy series of COVID-19-positive patients reveals that this disease, often conceptualized as a primarily respiratory viral illness, has widespread effects in the body including hypercoagulability, a hyperinflammatory state, and endothelial dysfunction. Targeting of these multisystemic pathways could lead to new treatment avenues as well as combination therapies against SARS-CoV-2 infection.
\end{abstract}

These authors contributed equally: Clare Bryce, Zachary Grimes, Elisabet Pujadas

Deceased November 15, 2020: Mary E. Fowkes

Supplementary information The online version contains supplementary material available at https://doi.org/10.1038/s41379021-00793-y.

$\triangle$ Carlos Cordon-Cardo

carlos.cordon-cardo@mssm.edu

1 Icahn School of Medicine at Mount Sinai, New York, NY, USA

2 University of Florida, Gainesville, FL, USA

\section{Introduction}

Severe acute respiratory syndrome coronavirus 2 (SARSCoV-2), the infective agent behind COVID-19, has rapidly spread around the globe resulting in substantial morbidity and mortality. As of 21 January, 2021, there have been 97.4 million confirmed cases globally including over 2 million deaths. In New York State alone, 1,279,811 cases and 41,587 deaths are documented [1].

Postmortem examinations, or autopsies, are the gold standard for the elucidation of the underlying pathophysiology of disease. Despite an explosion of literature addressing the clinical impact and molecular microbiology of SARS-CoV-2, autopsy studies remain underrepresented. Due to legitimate concerns about infection risk and limited personal protective equipment (PPE), early postmortem 
studies were initially either gross examination only, postmortem biopsies, restricted to certain organs, or case reports, with a few comprehensive series and some reviews emerging more recently [2-12].

In this comprehensive series, we report the findings from 100 consecutive autopsies of patients who died due to COVID-19 and summarize key histopathological findings within each organ system. We present the spectrum of our findings in the context of existing literature to advance the discussion of possible disease mechanisms.

\section{Methods}

\section{Laboratory studies and SARS-CoV-2 testing}

Premortem nasopharyngeal swab specimens were placed in universal transport media and tested by real-time reverse transcription polymerase chain reaction amplification on the fully automated sample-to-result single well double-target test cobas ${ }^{\circledR} 6800$ SARS-CoV-2 platform (Roche Molecular Systems, Branchburg, NJ).

\section{Autopsy and tissue collection}

Consented autopsies were performed at the Mount Sinai Hospital, which performs all autopsies for the Mount Sinai Health System's (MSHS) seven hospitals in the greater New York metropolitan area. The autopsies were conducted in a negative pressure room utilizing both PPE and techniques recommended from the current CDC Guidance for Postmortem Specimen Collection [13]. Extended collection procedures were utilized for autopsies with short postmortem intervals (PMIs) designated as rapid with a PMI of equal or less than $24 \mathrm{~h}$ [14-16].

\section{Special stains and immunostains}

Multiple unstained 3-5 $\mu \mathrm{m}$ thick sections were cut for special stains and immunohistochemistry (IHC). Special stains were applied according to standard protocols. Antibodies employed for IHC are listed in the Supplementary Methods Table. IHC staining was performed on a Leica Bond III automated stainer except for ACE-II, which was stained on the Discovery Ultra VENTANA systems (Roche).

\section{Electron microscopy}

Postmortem specimens for electron microscopy were placed in $3 \%$ buffered glutaraldehyde. Following postfixation in $1 \%$ osmium tetroxide, tissues were serially dehydrated and embedded in epoxy resin in standard fashion. One-micron toluidine-stained scout sections were prepared for light microscopic orientation; $80 \mathrm{~nm}$ ultrathin sections for EM were stained with uranyl acetate and lead citrate and examined in a Hitachi 7650 transmission electron microscope at $80 \mathrm{kV}$.

\section{Results}

\section{Patient characteristics and laboratory data}

The MSHS started performing autopsies on COVID-19positive patients on 20 March, 2020, with a total of 102 completed by 23 June, 2020. Two cases were restricted to no education or research (excluded from this study), one case was restricted to "no heart or kidney" and one to "brain only."

Patients' ages ranged from 29 to 94 years (median 68), with both a mean and median age of death in the mid-60s. Deaths occurred over every decade of adult life: 1 (20-29); 6 (30-39); 9 (40-49); 15 (50-59); 26 (60-69); 22 (70-79); 15 (80-89); and 6 (90-94), correlating with the breakdown of age for more than 400 COVID-19 deaths at MSHS. These patients exhibited a range of preexisting conditions and symptom profiles, including hypertension $62 \%$, diabetes mellitus $43 \%$, coronary artery disease $32 \%$, chronic kidney disease $30 \%$, asthma $18 \%$, heart failure $13 \%$, atrial fibrillation $10 \%$, obesity $11 \%$, coinfections $14 \%$, cancer $7 \%$, transplantation $6 \%$, and chronic obstructive pulmonary disease $5 \%$. A total of 63 patients were intubated, and 29 had health care directives not to intubate; the median time to intubation was 3 days, and the average time to intubation was 7 days. The average time to death from admission was 14 days (range of 0-87 days) (Supplementary Results Table 1).

Laboratory values for patients were compiled and were consistent with those previously reported (Supplementary Results Table 1) [17-19]. Markers relating to inflammation, including ferritin (mean at peak of 4384; ref 30-400 ng/ml), C-reactive protein (CRP) (mean at peak of 249; ref 0.0-5.0 $\mathrm{mg} / \mathrm{l}$ ), procalcitonin (mean at peak of 24.9 ; ref $<0.49 \mathrm{ng} /$ $\mathrm{ml}$ ), and white blood cell count (mean at peak of 19.1, ref. $\left.1.90-8.00 \times 10^{3} / \mu \mathrm{l}\right)$ composed predominantly of neutrophils (mean at peak neutrophil to lymphocyte ratio of 40.9), were elevated above normal reference values. The absolute lymphocyte count was mildly decreased (mean at nadir 0.9 ; ref $1.0-4.5 \times 10^{3} / \mu \mathrm{l}$ ). Cytokines IL-6 (mean at peak of 749.2; ref $0.0-5.0 \mathrm{pg} / \mathrm{ml}$ ), IL-8 (mean at peak of 162.4; ref $0.0-5.0 \mathrm{pg} / \mathrm{ml}$ ), and $\mathrm{TNF} \alpha$ (mean at peak of 40.0; ref $0.0-22.0 \mathrm{pg} / \mathrm{ml}$ ) were elevated, with cytokine IL-1b remaining within the reference range for most patients (mean at peak 1.8; ref $0.0-5.0 \mathrm{pg} / \mathrm{ml}$ ).

Anticoagulation therapy was given to $82 \%$ of patients. D-dimer levels were consistently elevated (mean at peak of 
$10.4 \mu \mathrm{g} / \mathrm{ml}$; ref $0.00-0.50 \mu \mathrm{g} / \mathrm{ml}$ ) despite administration of anticoagulation therapy. Fibrinogen was also elevated (mean at peak of 688 ; ref $175-450 \mathrm{mg} / \mathrm{dl}$ ). Thrombocytopenia was uncommon (platelet mean minimum of 164,000; ref $150,000-450,000 / \mu \mathrm{l})$. Elevations were seen in prothrombin time (mean at peak of 22.1; ref 12.3-14.9 s), partial thromboplastin time (mean at peak of 81.6; ref 25.4-34.9 s), and international normalized ratio (mean peak of 2.7). While these could partially be explained by the presence of anticoagulant therapy administered during most of these patients' admissions, these values were also elevated outside of the normal range at presentation in some patients who were not previously treated with anticoagulants.

\section{Autopsy findings}

Histologic sampling was performed on all 100 cases, including 35 rapid cases with a PMI of $24 \mathrm{~h}$ or less. Where the PMI was prolonged, tissue stock was saved; however, sampling was more limited due to severe autolysis. Consent restrictions or tissue autolysis resulted in the following total number of cases reviewed for each organ system below and in Table 1.

\section{Pulmonary system}

Right lung weight averaged $934 \mathrm{~g}$ (range: 300-1800 g; ref. range: $360-570 \mathrm{~g}$ ) and left lung weight averaged $1000 \mathrm{~g}$ (range: $300-1900 \mathrm{~g}$; ref. range: $325-480 \mathrm{~g}$ ). Grossly, the lungs showed either patchy areas of firm tan consolidation (Fig. 1A), generally with abundant edema fluid, or were diffusely firm and solid. Multiple cavitary lesions corresponding to severe necrotizing pneumonia were present in ten cases. Large pulmonary emboli obstructing the main pulmonary arteries were identified in six cases.

The lungs were histologically evaluated in 99 cases. The findings of 25 cases were previously reported in the series of Borczuk et al. [9]. The number of slides evaluated from each case ranged from 5 to 33, sampling each lobe in both peripheral and central locations. Histologically, the primary finding in the lung parenchyma was diffuse alveolar damage (DAD), which was seen in 82 cases. Of these, 54 were in the acute/early organizing phase and 28 showed more extensive organization. There was a statistically significant difference in the length of time between onset of symptoms and death between early/acute DAD (median $=11$ days) and organizing DAD (median $=26$ days; $p=0.0016$ ). Hyaline membranes (Fig. 1B, C) were variably present in all DAD cases, ranging from diffuse involvement of all sections to focal involvement of one or two sections. Edema and congestion/fresh blood were present to some extent in all cases. Associated type 2 pneumocyte hyperplasia was seen to some degree in all cases with DAD, although the
Table 1 Clinical and laboratory data summary.

\begin{tabular}{|c|c|c|c|}
\hline \multicolumn{4}{|l|}{ Pulmonary } \\
\hline \multirow[t]{7}{*}{ Lungs $(n=99)$} & Diffuse alveolar damage & $82 / 99$ & $83 \%$ \\
\hline & Early/acute & $54 / 82$ & $66 \%$ \\
\hline & Organizing & $28 / 82$ & $34 \%$ \\
\hline & Fibrotic & $0 / 82$ & $0 \%$ \\
\hline & Superimposed acute pneumonia & $45 / 82$ & $55 \%$ \\
\hline & Septal capillary proliferation & $19 / 99$ & $19 \%$ \\
\hline & Pulmonary thromboemboli & $6 / 99$ & \\
\hline \multicolumn{4}{|l|}{ Hematopoietic } \\
\hline \multirow[t]{7}{*}{ Spleen $(n=86)$} & Red pulp necrosis & 9/86 & $10 \%$ \\
\hline & Red pulp congestion & $24 / 86$ & $28 \%$ \\
\hline & Red pulp hemorrhage & $8 / 86$ & $9 \%$ \\
\hline & Germinal centers absent & $86 / 86$ & $100^{\circ}$ \\
\hline & Microthrombi & 3/86 & $3 \%$ \\
\hline & Extramedullary megakaryocytes & 2/86 & $2 \%$ \\
\hline & Hemophagocytic histiocytes & $11 / 86$ & $13 \%$ \\
\hline \multirow[t]{7}{*}{ Lymph nodes $(n=60)$} & Necrosis & $3 / 60$ & $5 \%$ \\
\hline & Vascular congestion & $23 / 60$ & $38 \%$ \\
\hline & Vascular transformation of sinuses & $8 / 60$ & $13 \%$ \\
\hline & Hemorrhage & $7 / 60$ & $12 \%$ \\
\hline & Germinal centers absent & $52 / 60$ & $87 \%$ \\
\hline & Microthrombi & $3 / 60$ & $5 \%$ \\
\hline & Extramedullary megakaryocytes & $2 / 60$ & $3 \%$ \\
\hline \multirow[t]{4}{*}{ Bone marrow $(n=11)$} & Hemophagocytosis & $8 / 11$ & $73 \%$ \\
\hline & Left shift granulopoiesis & $4 / 11$ & $36 \%$ \\
\hline & Decreased erythroid precursors & $1 / 11$ & $1 \%$ \\
\hline & Decreased megakaryocytes & $1 / 11$ & $1 \%$ \\
\hline \multicolumn{4}{|l|}{ CNS } \\
\hline \multirow[t]{2}{*}{$\operatorname{Brain}(n=58)$} & Acute-subacute infarcts & $19 / 58$ & $33 \%$ \\
\hline & Microthrombi & $17 / 58$ & $29 \%$ \\
\hline Pituitary $(n=19)$ & Pituitary necrosis/infarct & $5 \%$ & $1 / 19$ \\
\hline \multicolumn{4}{|l|}{ Cardiovascular } \\
\hline \multirow[t]{7}{*}{ Heart $(n=97)$} & Cardiac enlargement & $86 / 97$ & $92 \%$ \\
\hline & Myocyte hypertrophy and interstitial fibrosis & 96/97 & $99 \%$ \\
\hline & Mild interstitial chronic inflammation & 4/97 & $4 \%$ \\
\hline & Subendocardial inflammation & 1/97 & $1 \%$ \\
\hline & Epicardial chronic inflammation & $31 / 97$ & $32 \%$ \\
\hline & Epicardial hemophagocytosis & 1/97 & $1 \%$ \\
\hline & Bacterial endocarditis & 2/97 & $2 \%$ \\
\hline \multicolumn{4}{|l|}{ Genitourinary } \\
\hline \multirow[t]{4}{*}{ Kidney $(n=94)$} & Hypertensive and diabetic changes & $19 / 94$ & $20 \%$ \\
\hline & Acute tubular injury & 23/94 & $24 \%$ \\
\hline & Calcium phosphate crystals distal tubules & $10 / 94$ & $11 \%$ \\
\hline & Thromboemboli & 2/94 & $2 \%$ \\
\hline Bladder $(n=27)$ & No significant pathology & & \\
\hline $\begin{array}{l}\text { Fallopian tubes and ovaries }(n \\
=21)\end{array}$ & No significant pathology & & \\
\hline Prostate $(n=16)$ & No significant pathology & & \\
\hline Uterus $(n=14)$ & No significant pathology & & \\
\hline Testes, seminal vesicles $(n=6)$ & No significant pathology & & \\
\hline \multicolumn{4}{|l|}{ Gastrointestinal } \\
\hline GI tract $(n=92)$ & No significant pathology & & \\
\hline \multicolumn{4}{|l|}{ Hepatobiliary } \\
\hline \multirow[t]{6}{*}{ Liver $(n=92)$} & Cirrhosis & $12 / 92$ & $13 \%$ \\
\hline & Fatty liver disease & 28/92 & $30 \%$ \\
\hline & Venous outflow obstruction & $41 / 92$ & $45 \%$ \\
\hline & Early organizing thrombi & & \\
\hline & Portal venules & $37 / 92$ & $40 \%$ \\
\hline & Terminal hepatic venules & $20 / 92$ & $22 \%$ \\
\hline \multicolumn{4}{|l|}{ Endocrine } \\
\hline Pancreas $(n=81)$ & $\begin{array}{l}\text { Islet cell depletion and amyloid (diabetic } \\
\text { change) }\end{array}$ & $1 / 81$ & $1 \%$ \\
\hline Thyroid $(n=44)$ & Lymphocytic thyroiditis & $1 / 44$ & $2 \%$ \\
\hline Adrenal glands $(n=58)$ & No significant pathology & & \\
\hline
\end{tabular}

The time in days from symptom onset to death was significantly different between early/acute (median $=11$ days) and organizing $($ median $=26$ days $)$ DAD cases $(p$ value $=0.0016)$. 

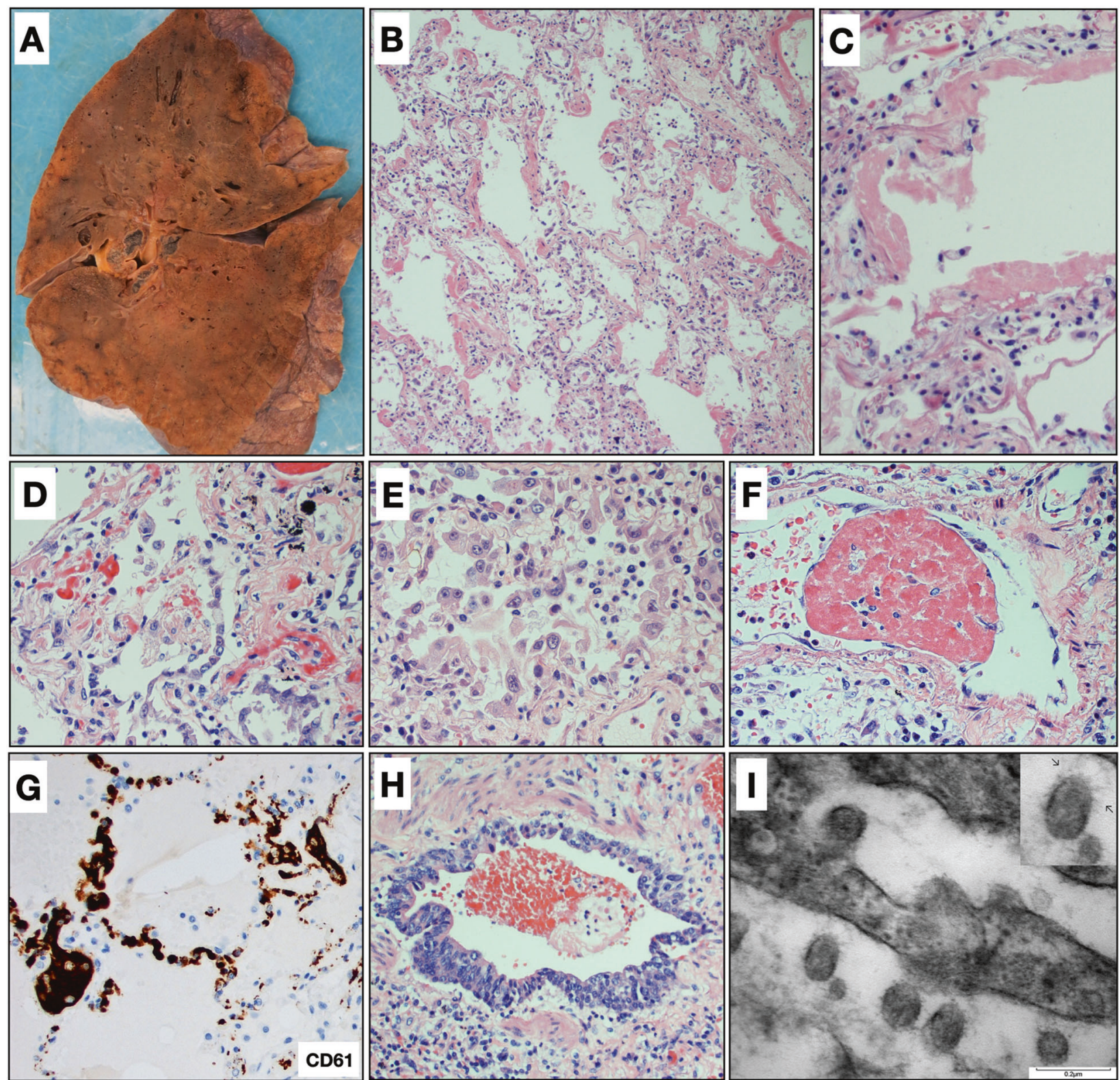

Fig. 1 Pulmonary findings. A Gross image of lung showing patchy areas of consolidation that corresponded to diffuse alveolar damage histologically. B Diffuse alveolar damage with prominent hyaline membranes and pneumocyte hyperplasia $(\mathrm{H} \& \mathrm{E}, \times 200)$. C Hyaline membrane lining an alveolar space with alveolar walls showing sparse chronic inflammation $(\mathrm{H} \& \mathrm{E}, \times 400)$. D Pneumocyte hyperplasia with relatively bland cytologic features $(\mathrm{H} \& \mathrm{E}, \times 400)$. E Pneumocyte hyperplasia with marked pleomorphism and cytologic atypia with occasional macronucleoli. Lymphocytes and macrophages are also present in the airspace lumens (H\&E, $\times 400)$. F Fibrin thrombi were observed in blood vessels in 34 cases $(\mathrm{H} \& \mathrm{E}, \times 600)$. G Platelet thrombi

degree of proliferation was variable and was greater in cases with more extensive disease. The cytologic features of pneumocyte proliferation were also variable. While some cases showed relatively bland features, others showed large pleomorphic cells, occasionally with multinucleation or prominent nucleoli. The latter features were typically found are highlighted by CD61 staining $(\mathrm{CD} 61, \times 400)$. H Bronchioles with regenerative basal cell hyperplasia and loss of cilia, consistent with prior injury $(\mathrm{H} \& \mathrm{E}, \times 400)$. I Electron micrograph from lung tissue portraying presence of multiple spherical virus particles showing spike-like electron-dense peplomeric projections ranging from 100 to $140 \mathrm{~nm}$. Note the attachment/budding of viral-like particles to the respiratory epithelium signaling possible endo/exocytosis as well as presence of extracellular virus particles. Scale bar, $0.2 \mu \mathrm{m}$. Insert: detail of a virion showing an electron-dense, relatively thick envelope with distinctive peplomers projecting from the surface giving the appearance of a solar corona.

in cases with diffuse disease (Fig. 1D, E). Squamous metaplasia was present in a minority of cases, usually focally. The interstitium typically contained varying amounts of lymphocytic inflammation that was generally mild and tended to be more diffuse in cases with extensive disease. A panel of immunostains performed on four cases 
demonstrated that the infiltrates were primarily $\mathrm{CD}_{4}^{+}$ $\mathrm{T}$ cells and $\mathrm{CD}_{163^{+}}$macrophages admixed with smaller numbers of $\mathrm{CD}^{+} \mathrm{T}$ cells and $\mathrm{CD} 20^{+} \mathrm{B}$ cells. Airspaces also contained occasional areas of lymphocyte and macrophage accumulation, which tended to increase with more extensive disease. Organizing pneumonia or organizing intra-alveolar fibrin was variably present in a minority of cases. No cases had histology consistent with acute fibrinous and organizing pneumonia or cryptogenic organizing pneumonia. Superimposed acute pneumonia was seen in 45 cases and was extensive and necrotizing in 10, including two cases with invasive Aspergillus. Intravascular fibrin thrombi were observed in 34 cases within small arteries or arterioles (Fig. 1F). Platelet aggregates and/or thrombi were difficult to appreciate on H\&E stained sections, usually corresponding to eosinophilic granular material in the vessels, but CD61 stains performed on 23 of the cases highlighted aggregates or thrombi in small arteries, arterioles, and capillaries in all but two cases on which stains were performed. Fibrin thrombi, when present, were observed only focally while platelet aggregates and thrombi tended to be present more frequently but were only prominent in one case, which demonstrated clusters of platelet thrombi in several areas (Fig. 1G). An additional interesting finding was the presence of capillary proliferation with inflammation and injury resembling endotheliitis in 19 cases. In regard to airway changes, the epithelium of the mainstem bronchi was largely denuded and the airways otherwise showed only minimal lymphocytic inflammation. Ulceration and acute inflammation were seen in two cases. The bronchioles in most cases showed variable degrees of regenerative basal cell hyperplasia and occasional squamous metaplasia suggestive of prior injury (Fig. 1H). Electron microscopy studies revealed the presence of multiple spherical virus particles showing spike-like electrondense peplomeric projections ranging from 100 to $140 \mathrm{~nm}$ (Fig. 1I).

The 17 cases lacking DAD included 5 of the 6 cases with large pulmonary emboli, 4 cases with extensive acute pneumonia, 1 case with extensive metastatic carcinoma, and the remainder with extra-pulmonary causes of death.

\section{Hematolymphoid system}

Spleens from 86 cases, thoracic lymph nodes from 60 cases, and bone marrow from 11 cases (9 sternal; 2 calvarial) were microscopically evaluated. Extensive red pulp necrosis was seen in nine spleens; confluent or patchy necrosis was also identified in lymph nodes of three cases (Fig. 2A). In other cases, the presence of necrosis may have been obscured by supervening autolytic changes, which were present in many of the specimens, most significantly affecting the splenic red pulp. A proportion of the spleens ( 24 cases) showed red pulp congestion, with 8 showing frank hemorrhage.

Other findings in lymph nodes included vascular congestion (23 cases), with vascular transformation of sinuses ( 8 cases), and hemorrhage (7 cases). Sinus histiocytosis was present in 50 cases, many with foci of hemophagocytosis (34 cases) (Fig. 2B, C). Prominent immunoblasts were present within sinuses and dispersed within the paracortex of seven cases. Notably, germinal centers were absent in the white pulp of all examined spleens, and absent in lymph nodes from 52 of the 60 examined cases. Furthermore, where present, the germinal centers tended to be small, hypoplastic, and often sparsely distributed (Fig. 2D). Electron microscopy of a thoracic lymph node demonstrated coronavirus-induced organelle-like replicative structures consistent with double-membrane vesicles, and intracytoplasmic spherical shaped virus particles with characteristic electron-dense envelope and fine peplomeric projections (Fig. 2E, F). While lineage could not be definitively established due to imperfect preservation, the cells containing these structures were relatively large, were juxtaposed to basement membrane, and did not contain prominent lysosomes. Microthrombi were identified in six separate cases, three lymph nodes and three spleens (Fig. 2G, H). Extramedullary megakaryocytes were identified in two lymph nodes and two spleens, encompassing four separate cases, with one of the spleens also showing clusters of erythroid precursors.

Hemophagocytic histiocytes were identified in the spleen (11 of 86 cases) (Fig. 2I, J). A proportion of spleens showed significant diminution of white pulp; in 16 cases, white pulp comprised $10 \%$ or less of the area of the parenchyma. Features of the bone marrow included hemophagocytosis (eight cases) (Fig. 2K), left-shifted granulopoiesis (four cases), decreased erythroid precursors (one case), and decreased megakaryocytes (one case).

Immunohistochemical stains were performed on lymph nodes of six representative cases. In four cases, there were mildly to markedly diminished $\mathrm{CD}^{+} \mathrm{T}$ lymphocytes, with one case showing a concomitant decrease in B lymphocytes. The other two cases showed no overt loss of B or T lymphocytes. All cases showed predominance of $\mathrm{CD} 4^{+} \mathrm{T}$ lymphocytes over $\mathrm{CD}^{+} \mathrm{T}$ lymphocytes, with $\mathrm{CD} 4^{+}$to $\mathrm{CD}^{+}$ratios ranging from approximately $3: 1$ to $30: 1$ (Fig. 2L, M).

Immunohistochemical stains of six representative spleens showed normal distributions of $\mathrm{CD}^{+} \mathrm{T}$ lymphocytes and $\mathrm{CD} 20^{+} \mathrm{B}$ lymphocytes within the white pulp, with predominance of $\mathrm{CD}^{+}{ }^{+} \mathrm{T}$ lymphocytes over $\mathrm{CD}^{+} \mathrm{T}$ lymphocytes $\left(\mathrm{CD}^{+}\right.$to $\mathrm{CD}^{+}$ratios ranging from approximately $10: 1$ to $30: 1$, in five cases in which the ratio was assessed). 

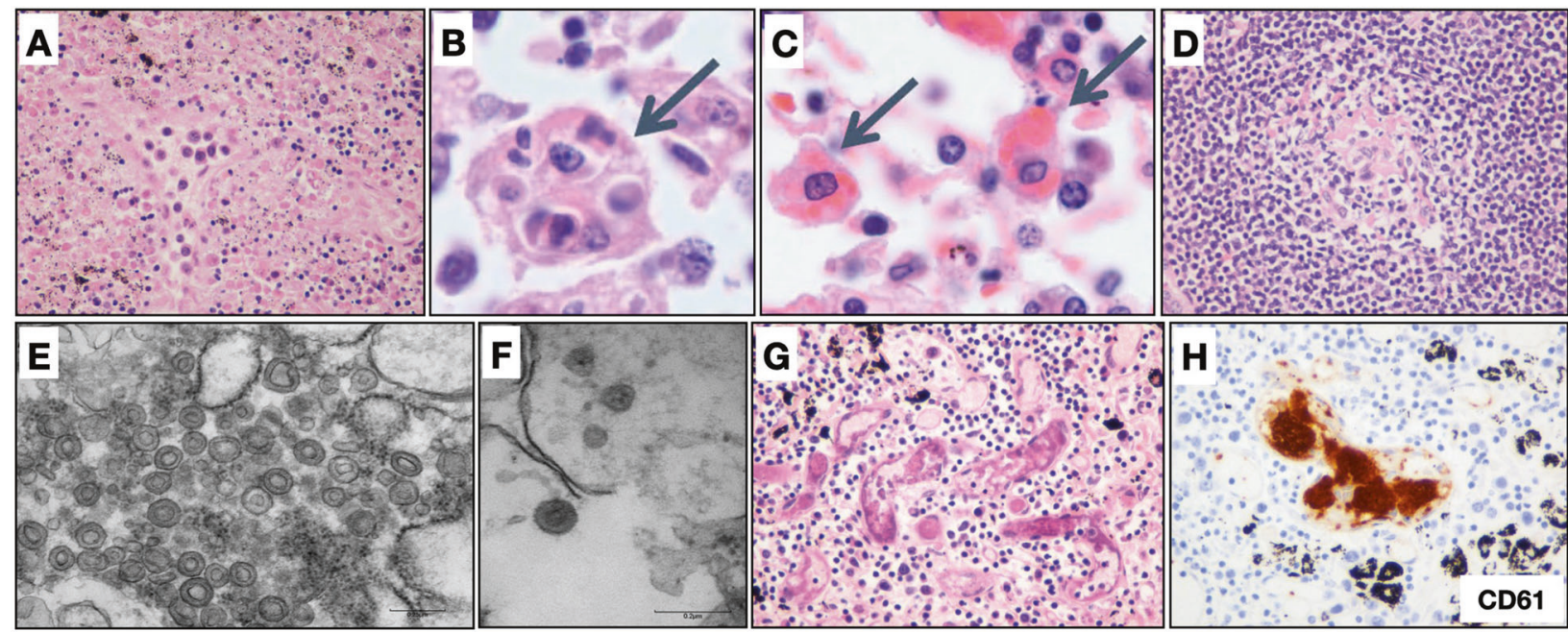

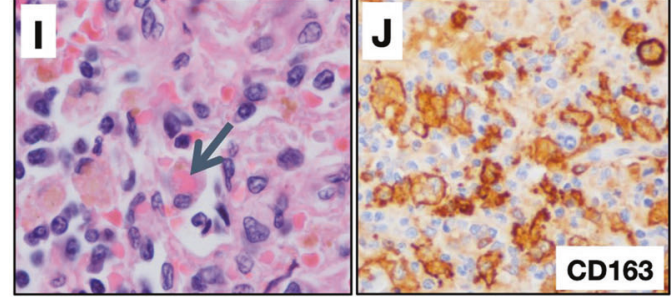

Fig. 2 Hematolymphoid system findings. A Lymph nodes with necrosis (H\&E, original magnification $\times 400$ ); B hemophagocytic histiocytes engulfing multiple cell types ( $\mathrm{H} \& \mathrm{E}$, original magnification $\times 1000)$ and $\mathbf{C}$ phagocytosing erythrocytes $(\mathrm{H} \& \mathrm{E}$, original magnification $\times 1000)$; and $\mathbf{D}$ follicle containing small, depleted, germinal center $(\mathrm{H} \& \mathrm{E}$, original magnification $\times 400)$. E Electron micrographs of a lymph node revealing coronavirus-induced organelle-like replicative structures consistent with double-membrane vesicles (DMVs), scale bar, $0.25 \mu \mathrm{m}$ and $\mathbf{F}$ intracytoplasmic spherically shaped virus particles with characteristic electron-dense envelope and fine peplomeric

\section{Central nervous system}

Neuropathological examination of 63 brains, with histologic evaluation of 58, revealed a range of abnormal pathology. The most frequent pathological findings were of acute/ early-subacute infarction, observed in 19 of 58 cases and the widespread associated presence of microthrombi, noted in 17 of 58 cases (Fig. 3A-F). At least one additional case showed areas of necrosis/infarction restricted to the pituitary ( 1 of 19 pituitaries examined). The vascular distribution of the acute infarcts was variable: one case showed a large cerebral artery territory infarct, but much more common were small and patchy microinfarcts located within the peripheral neocortex and the deeper gray matter structures, some of which were ischemic and others hemorrhagic. A notable and consistent abnormality was the presence of microthrombi often associated with small and patchy infarction, many less than $1 \mathrm{~mm}$ in size (Fig. 3A-F). Vascular congestion appeared out of proportion to what is typically seen, and was sometimes accompanied by acute parenchymal microhemorrhages, suggestive of vascular projections, scale bar $0.2 \mu \mathrm{m}$. G Lymph node with microthrombi $(\mathrm{H} \& \mathrm{E}$, original magnification $\times 400), \mathbf{H}$ with platelets highlighted by labeling for CD61 (original magnification $\times 400$ ). I Spleen with hemophagocytosis (H\&E, original magnification $\times 1000)$, J with splenic macrophages highlighted by labeling for CD163 (original magnification $\times 1000)$. K Bone marrow with hemophagocytosis $(\mathrm{H} \& \mathrm{E}$, original magnification $\times 1000$ ). $\mathbf{L}$ CD4 (original magnification $\times 100$ ) and M CD8 (original magnification $\times 100$ ) labeling in a lymph node demonstrating disproportionate loss of CD8+ T lymphocytes.

damage and reperfusion injury (Fig. 3B). Bacterial endocarditis was present in two cases, as well as subendocardial inflammation in one case (see "Cardiovascular system" below); these had associated multifocal cerebral infarcts/ lesions, without organisms on special stains. Of note, immunohistochemical staining revealed robust expression of the ACE2 receptor in intraparenchymal blood vessels (Fig. 3G; controls in Supplementary Figure).

In addition to the frequent manifestation of infarcts, one case (where autopsy also revealed acute endocarditis) demonstrated features most consistent with acute disseminated encephalomyelitis/acute hemorrhagic leukoencephalitis (ADEM/AHLE). Macroscopically, we observed innumerable punctate hemorrhagic-like lesions within the white matter (Fig. $3 \mathrm{H}$ ), which histologically manifested as numerous small foci of perivascular zones of demyelination (Fig. 3I), each generally less than $1 \mathrm{~mm}$ in size, some with mild focal acute perivenular inflammation and rare evidence for perivascular hemorrhagic necrosis (Fig. 3I).

The remainder of brains examined, representing the majority of cases, showed less striking histological 


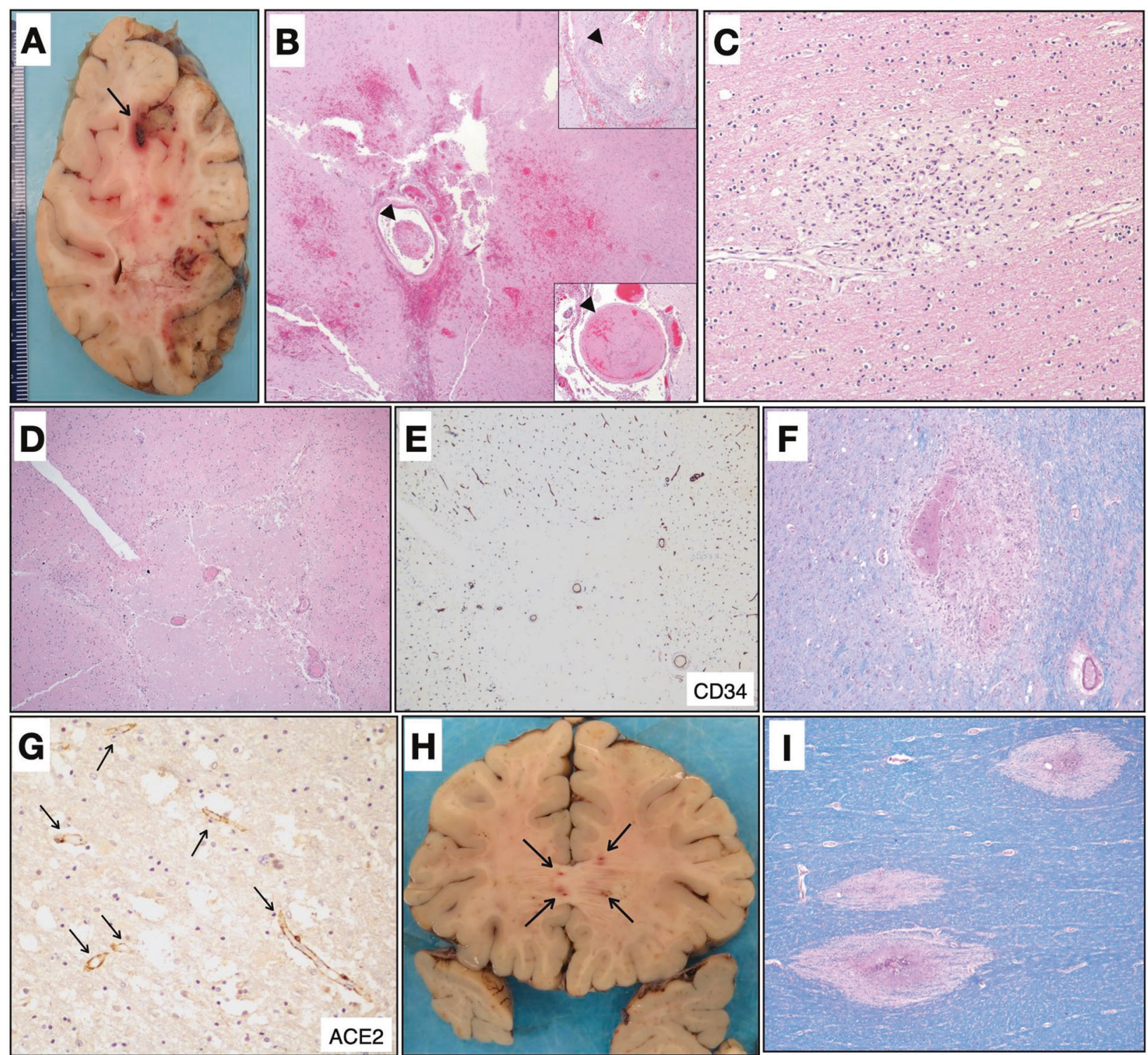

Fig. 3 Neuropathologic findings. A Coronal section of the right cerebral hemisphere containing multiple acute microinfarcts and focal region suggestive of a thrombosed and hemorrhagic blood vessel (arrow). B Histological confirmation of an acute infarct with scattered microhemorrhages and associated microthrombi (arrowheads). Insets: two additional identified microthrombi (arrowheads) (H\&E, $\times 400)$. C Subacute microinfarct with foamy macrophages $(H \& E, \times 100)$. D Acute ischemic infarct associated with mildly hyperplastic and congested blood vessels (H\&E, ×400). E Blood vessels are highlighted by
CD34 ( $\times 400)$. F Area of microhemorrhage with associated reactive changes within the basal ganglia (LFB, $\times 400$ ). G Immunohistochemical staining highlights ACE2 expression within many cerebral blood vessels (ACE2, $\times 100$, arrows). H Coronal section at the level of the genu of the corpus callosum showing areas of hemorrhage and myelin loss. I Histology of the corpus callosum lesions, demonstrating small areas of perivascular myelin loss, highlighted by luxol fast blue stain $(\mathrm{LFB})$, rarely associated with hemorrhage $(\times 400)$.

\section{Cardiovascular system}

All but 8 of 97 hearts examined had gross cardiac enlargement, with many having left ventricular hypertrophy and moderate to marked coronary atherosclerosis. All but one case revealed varying degrees of myocyte hypertrophy and interstitial fibrosis, consistent with preexisting hypertensive and/or atherosclerotic cardiovascular disease. In four cases, 
there was patchy mild interstitial chronic inflammation within the myocardium without associated myonecrosis, and in one additional case there was a surprising moderate subendocardial inflammatory infiltrate composed of predominantly $\mathrm{CD}^{+}{ }^{+} \mathrm{T}$ lymphocytes (both $\mathrm{CD} 4^{+}$and $\mathrm{CD} 8^{+}$) and a similar number of $\mathrm{CD} 163^{+}$macrophages with a smaller number of $\mathrm{CD} 20^{+}$B lymphocytes and a few scattered neutrophils. In 31 out of 97 cases, there was a slight patchy epicardial mononuclear infiltrate of predominantly $\mathrm{T}$ lymphocytes, but which was similarly seen in age- and sexmatched controls. In three cases, there were also small vessel thrombi in areas where there were epicardial inflammatory infiltrates, not seen in age- and sex-matched controls. In a single case, there was hemophagocytosis within an area of epicardial inflammation. There was no evidence of vasculitis in any of the cases. In two cases there was bacterial endocarditis.

COVID-19-related findings in the genitourinary, gastrointestinal, hepatobiliary, and endocrine systems were less striking and are described in the Supplementary Results section.

\section{Discussion}

COVID-19, the clinical syndrome caused by SARS-CoV-2, is increasingly recognized as a far more complex multiorgan and heterogeneous illness than initially anticipated. Some patients display a diverse array of symptoms and complications, including thromboembolic disease even in the setting of anticoagulant therapy [20], a hyperinflammatory state, and clinical courses complicated by abrupt, unexpected deterioration during the recovery phase. In addition, reports of morbidity in young patients [21] and children with Kawasaki-like disease [22] are emerging.

Our autopsy series underscores the multisystem involvement of COVID-19, although respiratory symptoms and findings still predominated. With the exception of 17 cases, all of the lungs showed DAD. Interestingly, four out of the six cases of PE occurred among the first 17 autopsies (performed on or before April 7, 2020), just before systemwide COVID-19 anticoagulation guidelines were issued at Mount Sinai on April 9, 2020. With the caveat that only a fraction of patients who died of COVID-19 were autopsied and that other factors may also be involved, it is possible that these guidelines may have contributed to a sharp decline in PE as a cause of mortality in COVID patients at our institution, which is consistent with retrospective analyses of larger patient cohorts [23].

DAD is the common end result of a myriad of pulmonary insults and is related to an underlying mechanism of alveolar and endothelial damage. Like the COVID-19 cases, DAD was also commonly reported in cases of Middle East respiratory syndrome coronavirus (MERS-CoV), SARS-CoV-1, and influenza [24-28]. Regardless of etiology, both type 1 pneumocytes and capillary endothelial cells are typically affected due to the extremely thin barrier between the two and the presence of a fused basement membrane. The pathogenesis is complex and numerous pathways are potentially involved [29]. From a pathologic standpoint, an etiology for DAD is usually not apparent from histologic findings alone. The histologic features identified in the COVID-19 cases in our series are typical of the range of findings reported in DAD, and are similar to those published in other reports or series of COVID-19 autopsies [4-7, 30-36]

The lack of prominent intra-alveolar fibrin in most cases in our series is in contrast to the findings reported in a component of SARS cases as well as in a postmortem biopsy study of COVID-19 cases [26, 35]. Whether this represents a different manifestation of disease in the SARS cases or a different form of disease in earlier COVID-19 cases, respectively, is unclear. Given the alterations in peripheral blood coagulation studies in many COVID patients, the finding of fibrin and platelet thrombi is of particular interest; however, both fibrin and platelet thrombi are a well-recognized finding in cases of DAD [37, 38], and coagulopathies and thrombi were also noted as a component of SARS-CoV-1 and MERS [39]. It has been suggested that the number of thrombi in COVID patients is greater than in non-COVID DAD, and the capillary findings are similarly more pronounced [5, 33, 40]. Capillary proliferations were observed in 19 of our cases and only in the setting of acute phase disease. The findings in our cases resembled those reported by Magro et al. [41], and we did not observe findings similar to those reported by De Michele, et al. [10]. Capillary proliferations have been reported in earlier descriptions of DAD and would be an expected finding in areas of ongoing repair following injury [37]. It is currently unclear whether either of these more recently reported vascular features corresponded to the findings reported by Ackermann et al. [40]. Review of six cases of non-COVID DAD from our autopsy files was performed, and they were similarly stained with CD61. The non-COVID cases did show fewer platelet thrombi in comparison to the COVID cases, as well as fewer fibrin thrombi and capillary proliferations; however, the cases were not controlled for degree of hyaline membrane formation or length of disease and some authors have contended that the histology of COVID-19 versus non-COVID DAD shows no significant differences [42]. Regardless, particularly in light of the findings of microthrombi in the other organ systems, including the brain, our observations support that coagulopathy and thrombosis are a significant component of COVID-19-related morbidity and mortality.

Review of hematolymphoid tissues showed histopathological features with possible correlations to COVID-19- 
related immune dysfunction. Notably, all spleens and a large majority of lymph nodes showed absence of germinal centers, and, where present, germinal centers tended to be small and hypoplastic. This finding aligns with those of Kaneko et al., who report absence of germinal centers in lymph nodes and spleens of COVID-19 patients, and propose that this is the consequence of cytokine-mediated disruption of BCL- $6^{+} \mathrm{T}$ follicular helper cell differentiation, and may underlie compromised humoral immunity associated with COVID-19 disease [43]. The majority of lymph nodes that were immunohistochemically assessed showed decreased $\mathrm{T}$ lymphocytes, with a disproportionate decrease in the $\mathrm{CD}^{+}$subset, and relative preservation of B lymphocytes. While acknowledging small sample size, this may be a histopathological analog to the decreased peripheral blood lymphocyte counts in our cohort, and as previously described in COVID-19 patients [44, 45]. Importantly, however, the above findings must be interpreted with the caveat that corticosteroid therapy may have contributed to lymphocyte depletion in many of the patients.

The identification of hemophagocytic histiocytes in lymph nodes, spleen, bone marrow, liver sinusoids, and epicardium is consonant with the clinical picture of a hyperinflammatory syndrome. However, the possibility of secondary hemophagocytic lymphohistiocytosis (sHLH) should only be invoked with caution, as presence of hemophagocytic histiocytes is not specific to this diagnosis, and may occur in other hyperinflammatory states such as sepsis [46]. While the cases under review did not necessarily meet criteria for diagnosis of hemophagocytic lymphohistiocytosis, as set forth by HLH-2004 guidelines [47], evaluation was incomplete, as for the majority of these patients, pertinent clinical data were lacking. While sHLH is a rare entity, in adults it is most commonly precipitated by viral infection [48], and is frequently associated with pulmonary complications, including acute respiratory distress syndrome (ARDS) [48]. It has been noted that cytokine abnormalities in COVID-19 patients in many respects parallel those associated with sHLH [49]. Furthermore, impairment of NK-cell function, a feature of primary HLH, is described in association with COVID-19 disease [50].

Clinically, the finding of elevated CRP, cytokines IL-6, IL-8, and TNF $\alpha$ in our patients are also consistent with a hyperinflammatory state, and are part of the mechanism of injury in ARDS and hypercoagulation [2, 29, 51]. Similar findings have been previously reported in SARS-CoV-1 and MERS, and have been more recently described in COVID19 patients [26, 52-54]. Among the altered cytokines, significantly elevated IL-6 is a poor prognostic marker in critically ill patients $[55,56]$.

Necrosis observed in subsets of the lymph nodes and spleens could potentially be attributed to viral cytopathic effect, although the possibility of an ischemic component cannot be excluded. Notably, electron microscopy of lymph node tissue from one case showed features suggestive of coronavirus replication in imperfectly preserved cells. Although the lineage of these cells could not be firmly established in view of the poor state of preservation, their size excludes lymphocytes, endothelial cells, and granulocytes, and absence of prominent lysosomes tends to exclude macrophages. In view of their juxtaposition to basement membrane, these could represent fibroblastic reticular cells (FRCs); however, definitive classification will require further investigation. FRCs have both a structural role, as well as a multiplicity of immunomodulatory functions in lymph nodes and other secondary lymphoid organs [57]; their infection, if confirmed, could contribute to immunological dysregulation in COVID-19 disease.

The major pathological findings in the CNS of COVID19 patients are related to the presence of multifocal infarction, with higher than expected population frequency [58] and atypical distribution, often associated with microthrombi. Comorbid conditions, while present in many of the cases, did not fully account for the type of infarcts seen and their distribution. Hypertension, the most critical risk factor for stroke [59], was present clinically and/or histologically in 49 of the 58 cases. Infarcts related to hypertensive vasculopathy typically vary in age and are restricted to the subcortical white matter and deep gray structures; they do not present as multiple and simultaneously occurring lesions of broad peripheral and deep gray distribution, as noted herein. Diabetes mellitus, clinically present in five cases with acute infarcts, is associated with accelerated atherosclerosis and small vessel ischemic disease [60], but similarly would not be expected to be associated with multifocal lesions of similar age in such a broad vascular distribution. Hypertensive vasculopathy, diabetes [61], and cerebral amyloid angiopathy typically result in hemorrhagic infarcts/ cerebral hemorrhages; in contrast, COVID-19 infarcts were more often ischemic than hemorrhagic. Rather, the foci of hemorrhage seen in COVID-19 cases appeared to be secondary to reperfusion injury, with focal punctate hemorrhages found within larger areas of necrosis. Given the severe respiratory injury in COVID-19 cases, brain infarcts secondary to low oxygenation would be suspected, with a typically large and confluent infarction in a watershed arterial flow territory. Such infarcts were seen in 2 of the 19 cases with acute infarction. Similarly, global hypoxia with obvious diffuse swelling was seen in only four cases.

The appearance of microinfarcts in COVID-19 cases was most reminiscent of thromboembolic etiology. Atherosclerotic or thromboembolic infarcts originating from embolic fragmentation of large proximal atheromatous plaque, atrial fibrillation, or endocarditis can cause multifocal acute infarcts in a distinct pattern similar to what was seen with the examined COVID-19 cases. Atrial fibrillation 
is one of the most common conditions responsible for multifocal acute embolic infarcts. However, despite hospitalization and close monitoring, atrial fibrillation was only identified in two COVID-19 cases with acute infarction. Only two cases of endocarditis were identified in our cohort. Mycotic emboli can result in multifocal infarcts, similar to endocarditis, but only one case showed organisms on special stains and was associated with invasive pulmonary aspergillosis and diffuse anoxic brain injury.

Overall, the increased incidence and pattern of multifocal acute infarcts and numerous microthrombi, in both venous and arterial vessels in a somewhat unusual distribution, raises the possibility of unique vascular pathology related to COVID-19. These findings conform with reported increased risk for cryptogenic stroke in COVID-19 patients identified by imaging studies [62] and sometimes too small for MRI detection [63] and could explain some of the clinical neuropsychiatric abnormalities seen in COVID-19 patients, such as confusion and mental status change, as well as the still illdefined post-COVID neurological sequelae beginning to emerge. Interestingly, immunohistochemical evaluation of ACE2 in the brain demonstrates an ACE2-positive phenotype in the endothelial cells of intraparenchymal blood vessels, while the brain parenchyma itself does not show significant staining for ACE2. In conjunction with the prominent vascular findings seen in our patients, this suggests that the endothelial cells themselves may be targeted by the virus and mediate the vascular and neuropsychiatric symptoms seen in COVID-19 patients in absence of encephalitis.

In addition to infarction, one case also showed evidence for perivenular demyelination focally associated with necrosis, most consistent with ADEM and/or its hyperacute form, AHLE. ADEM/AHLE is presumed to be an autoimmune disorder which usually occurs following an infection, most common in males and children or young adults [64]. Several studies have now reported on the rare occurrence of an ADEM-like illness in COVID-19 patients [6569], consistent with our findings.

Postmortem studies are prone to some limitations, perhaps the most important of which is the inability to assess illness dynamics, as the evaluation takes place at the end of the disease course. Tissue preservation is often a concern as well, mitigated in part by the large number of rapid autopsies in our series. In addition, the complexity of disease processes within this cohort (coinfections, organ transplant, and malignancy) makes generalization about COVID-19-related findings somewhat difficult.

Overall, our postmortem study of a large cohort of COVID-19 patients emphasizes the multisystem effects of COVID-19 infection, notably the role of extensive coagulopathy and a hyperinflammatory state. Ongoing and future studies targeting these pathways could potentially lead to a shift in therapeutic strategies.

\section{Data availability}

The datasets used and/or analyzed during the current study are available from the corresponding author on reasonable request.

Acknowledgements This manuscript is dedicated to the memory of Mary E. Fowkes, MD, PhD, whose leadership, vision, and dedication made this work possible. She will be missed as a colleague, mentor, professional, and, most of all, as a friend. The authors thank Valentín Fuster for his guidance and advice. The authors thank Etty Cortes for her help with tissue banking. The authors thank Florian Krammer, Fatima Amanat, Akm Juber Ahmed, Jin Xu, Alex Fayad, Monika Garcia-Barros, Sara Olson, Rachel Olivares, Tin Htwe Thin, and Frances Avila for their experimental support. The authors thank Ian Hsu for helpful discussion.

Author contributions $\mathrm{MEF}, \mathrm{CCC}, \mathrm{CB}, \mathrm{ZG}, \mathrm{EP}, \mathrm{SA}, \mathrm{MBB}, \mathrm{BP}, \mathrm{FS}$, MIF, NH, SMEJ, NMT, AG-S, and AP-M: developed the study concept and design. MEF, CCC, CB, ZG, EP, SA, MBB, MAR, GKH, AC, MU, REG, JR, BP, FS, MIF, NH, RD, SMEJ, NMT, RS, AF, and AP-M: development of methodology and writing and review and revision of the paper. MEF, CCC, CB, ZG, EP, SA, MBB, TH, AS, ZZ, JC, MAR, GKH, MU, AC, REG, JR, BP, FS, MIF, SMEJ, NMT, ZM, BV, RB, NH, RD, PS, CK, JC, IM, K-LW, RS, JJ, JL, and AP-M: acquisition, analysis and interpretation of data, and statistical analysis. All other authors provided technical and material support.

Funding No external funding was used in this study.

\section{Compliance with ethical standards}

Conflict of interest The authors declare no competing interests.

Consent to participate All autopsies were done with written consent from the legal next of kin.

Ethics approval The Icahn School of Medicine Institution Review Board considers autopsies as nonhuman subjects (decedents). Approval for this study was provided by the Icahn School of Medicine Grants and Contracts Office.

Publisher's note Springer Nature remains neutral with regard to jurisdictional claims in published maps and institutional affiliations.

\section{References}

1. COVID-19 Dashboard by the Center for Systems Science and Engineering (CSSE) at Johns Hopkins University, [Internet]. [Accessed 21 Jan 2021]. Available from https://coronavirus.jhu. edu.

2. Xu Z, Shi L, Wang Y, Zhang J, Huang L, Zhang C, et al. Pathological findings of COVID-19 associated with acute respiratory distress syndrome. Lancet Respir Med. 2020;8:420-2.

3. Carsana L, Sonzogni A, Nasr A, Rossi RS, Pellegrinelli A, Zerbi $\mathrm{P}$, et al. Pulmonary post-mortem findings in a series of COVID-19 cases from northern Italy: a two-centre descriptive study. Lancet Infect Dis. 2020;20:1135-40.

4. Edler C, Schroder AS, Aepfelbacher M, Fitzek A, Heinemann A, Heinrich F, et al. Dying with SARS-CoV-2 infection-an autopsy study of the first consecutive 80 cases in Hamburg, Germany. Int J Leg Med. 2020;134:1275-84. 
5. Fox SE, Akmatbekov A, Harbert JL, Li G, Quincy Brown J, Vander Heide RS. Pulmonary and cardiac pathology in African American patients with COVID-19: an autopsy series from New Orleans. Lancet Respir Med. 2020;8:681-6.

6. Martines RB, Ritter JM, Matkovic E, Gary J, Bollweg BC, Bullock $\mathrm{H}$, et al. Pathology and pathogenesis of SARS-CoV-2 associated with fatal coronavirus disease, United States. Emerg Infect Dis. 2020;26:2005-15.

7. Wu JH, Li X, Huang B, Su H, Li Y, Luo DJ, et al. [Pathological changes of fatal coronavirus disease 2019 (COVID-19) in the lungs: report of 10 cases by postmortem needle autopsy]. Zhonghua Bing Li Xue Za Zhi. 2020;49:568-75.

8. Maiese A, Manetti AC, La Russa R, Di Paolo M, Turillazzi E, Frati P, et al. Autopsy findings in COVID-19-related deaths: a literature review. Forensic Sci Med Pathol. 2020. https://doi.org/ 10.1007/s12024-020-00310-8.

9. Borczuk AC, Salvatore SP, Seshan SV, Patel SS, Bussel JB, Mostyka M, et al. COVID-19 pulmonary pathology: a multiinstitutional autopsy cohort from Italy and New York City. Mod Pathol. 2020;33:2156-68.

10. De Michele S, Sun Y, Yilmaz MM, Katsyv I, Salvatore M, Dzierba AL, et al. Forty postmortem examinations in COVID-19 patients. Am J Clin Pathol. 2020;154:748-60.

11. Elsoukkary SS, Mostyka M, Dillard A, Berman DR, Ma LX, Chadburn A, et al. Autopsy findings in 32 patients with COVID19: a single-institution experience. Pathobiology. 2021;88:56-68.

12. Borczuk AC. Pulmonary pathology of COVID-19: a review of autopsy studies. Curr Opin Pulm Med. 2021. Epub ahead of print. PMID 33399353

13. Collection and Submission of Postmortem Specimens from Deceased Persons with Confirmed or Suspected COVID-19. Postmortem guidance, [Internet], Center for Disease Control and Prevention. [Accessed 6 May 2020]. Available from https://www. cdc.gov/coronavirus/2019-ncov/hcp/guidancepostmortemspecimens.html.

14. Pisapia DJ, Salvatore S, Pauli C, Hissong E, Eng K, Prandi D, et al. Next-generation rapid autopsies enable tumor evolution tracking and generation of preclinical models. JCO Precis Oncol. 2017;2017:1-13.

15. Krook MA, Chen HZ, Bonneville R, Allenby P, Roychowdhury S. Rapid research autopsy: piecing the puzzle of tumor heterogeneity. Trends Cancer. 2019;5:1-5.

16. Lange KR, Fischer C, Rajappa P, Connors S, Pisapia D, Greenfield JP, et al. Rapid autopsy of a patient with recurrent anaplastic ependymoma. Palliat Support Care. 2018;16:238-42.

17. Zhou F, Yu T, Du R, Fan G, Liu Y, Liu Z, et al. Clinical course and risk factors for mortality of adult inpatients with COVID-19 in Wuhan, China: a retrospective cohort study. Lancet. 2020;395: 1054-62.

18. Zhang Y, Xiao M, Zhang S, Xia P, Cao W, Jiang W, et al. Coagulopathy and antiphospholipid antibodies in patients with Covid-19. N Engl J Med. 2020;382:e38.

19. Connors JM, Levy JH. COVID-19 and its implications for thrombosis and anticoagulation. Blood. 2020. https://doi.org/10. 1182/blood.2020006000.

20. Klok FA, Kruip M, van der Meer NJM, Arbous MS, Gommers D, Kant KM, et al. Incidence of thrombotic complications in critically ill ICU patients with COVID-19. Thromb Res. 2020. https:// doi.org/10.1016/j.thromres.2020.04.013.

21. Oxley TJ, Mocco J, Majidi S, Kellner CP, Shoirah H, Singh IP, et al. Large-vessel stroke as a presenting feature of Covid-19 in the young. N Engl J Med. 2020. https://doi.org/10.1056/NEJMc2009787.

22. Jones VG, Mills M, Suarez D, Hogan CA, Yeh D, Bradley Segal J, et al. COVID-19 and Kawasaki disease: novel virus and novel case. Hosp Pediatr. 2020. https://doi.org/10.1542/hpeds.2020-0123.
23. Nadkarni GN, Lala A, Bagiella E, Chang HL, Moreno PR, Pujadas E, et al. Anticoagulation, bleeding, mortality, and pathology in hospitalized patients with COVID-19. J Am Coll Cardiol. 2020;76:1815-26.

24. Alsaad KO, Hajeer AH, Al Balwi M, Al Moaiqel M, Al Oudah N, Al Ajlan A, et al. Histopathology of Middle East respiratory syndrome coronovirus (MERS-CoV) infection-clinicopathological and ultrastructural study. Histopathology. 2018;72:516-24.

25. Gill JR, Sheng ZM, Ely SF, Guinee DG, Beasley MB, Suh J, et al. Pulmonary pathologic findings of fatal 2009 pandemic influenza A/H1N1 viral infections. Arch Pathol Lab Med. 2010;134: 235-43.

26. Hwang DM, Chamberlain DW, Poutanen SM, Low DE, Asa SL, Butany J. Pulmonary pathology of severe acute respiratory syndrome in Toronto. Mod Pathol. 2005;18:1-10.

27. Nicholls JM, Poon LL, Lee KC, Ng WF, Lai ST, Leung CY, et al. Lung pathology of fatal severe acute respiratory syndrome. Lancet. 2003;361:1773-8.

28. Voltersvik P, Aqrawi LA, Dudman S, Hungnes O, Norwegian Lung Pathology G, Bostad L, et al. Pulmonary changes in Norwegian fatal cases of pandemic influenza H1N1 (2009) infection: a morphologic and molecular genetic study. Influenza Other Respir Viruses. 2016;10:525-31.

29. Huppert LA, Matthay MA, Ware LB. Pathogenesis of acute respiratory distress syndrome. Semin Respir Crit Care Med. 2019;40:31-39.

30. Tian S, Xiong Y, Liu H, Niu L, Guo J, Liao M, et al. Pathological study of the 2019 novel coronavirus disease (COVID-19) through postmortem core biopsies. Mod Pathol. 2020. https://doi.org/10. 1038/s41379-020-0536-x.

31. Barton LM, Duval EJ, Stroberg E, Ghosh S, Mukhopadhyay S. COVID-19 autopsies, Oklahoma, USA. Am J Clin Pathol. 2020;153:725-33.

32. Bosmuller H, Traxler S, Bitzer M, Haberle H, Raiser W, Nann D, et al. The evolution of pulmonary pathology in fatal COVID-19 disease: an autopsy study with clinical correlation. Virchows Arch. 2020. https://doi.org/10.1007/s00428-020-02881-x.

33. Carsana L, Sonzogni A, Nasr A, Rossi RS, Pellegrinelli A, Zerbi $\mathrm{P}$, et al. Pulmonary post-mortem findings in a series of COVID-19 cases from northern Italy: a two-centre descriptive study. Lancet Infect Dis. 2020. https://doi.org/10.1016/S1473-3099(20)30434-5.

34. Nunes Duarte-Neto A, de Almeida Monteiro RA, da Silva LFF, Malheiros D, de Oliveira EP, Theodoro Filho J, et al. Pulmonary and systemic involvement of COVID-19 assessed by ultrasoundguided minimally invasive autopsy. Histopathology. 2020. https:// doi.org/10.1111/his. 14160 .

35. Copin MC, Parmentier E, Duburcq T, Poissy J, Mathieu D, Lille C-I, et al. Time to consider histologic pattern of lung injury to treat critically ill patients with COVID-19 infection. Intensive Care Med. 2020;46:1124-6.

36. Wichmann D, Sperhake JP, Lutgehetmann M, Steurer S, Edler C, Heinemann A, et al. Autopsy findings and venous thromboembolism in patients with COVID-19. Ann Intern Med. 2020. https:// doi.org/10.7326/M20-2003.

37. Tomashefski JF Jr., Davies P, Boggis C, Greene R, Zapol WM, Reid LM. The pulmonary vascular lesions of the adult respiratory distress syndrome. Am J Pathol. 1983;112:112-26.

38. Tomashefski JF Jr. Pulmonary pathology of acute respiratory distress syndrome. Clin Chest Med. 2000;21:435-66.

39. Giannis D, Ziogas IA, Gianni P. Coagulation disorders in coronavirus infected patients: COVID-19, SARS-CoV-1, MERSCoV and lessons from the past. J Clin Virol. 2020;127:104362.

40. Ackermann M, Verleden SE, Kuehnel M, Haverich A, Welte T, Laenger F, et al. Pulmonary vascular endothelialitis, thrombosis, and angiogenesis in Covid-19. N Engl J Med. 2020;383:120-8. 
41. Magro C, Mulvey JJ, Berlin D, Nuovo G, Salvatore S, Harp J, et al. Complement associated microvascular injury and thrombosis in the pathogenesis of severe COVID-19 infection: a report of five cases. Transl Res. 2020;220:1-13.

42. Konopka KE, Nguyen T, Jentzen JM, Rayes O, Schmidt CJ, Wilson AM, et al. Diffuse alveolar damage (DAD) from coronavirus disease 2019 infection is morphologically indistinguishable from other causes of DAD. Histopathology. 2020. https://doi.org/10.1111/his.14180.

43. Kaneko N, Kuo HH, Boucau J, Farmer JR, Allard-Chamard H, Mahajan VS, et al. Loss of Bcl-6-expressing T follicular helper cells and germinal centers in COVID-19. Cell. 2020;183:143-57.

44. Wang F, Nie J, Wang H, Zhao Q, Xiong Y, Deng L, et al. Characteristics of peripheral lymphocyte subset alteration in COVID-19 pneumonia. J Infect Dis. 2020. https://doi.org/10. 1093/infdis/jiaa150.

45. Chen N, Zhou M, Dong X, Qu J, Gong F, Han Y, et al. Epidemiological and clinical characteristics of 99 cases of 2019 novel coronavirus pneumonia in Wuhan, China: a descriptive study. Lancet. 2020;395:507-13.

46. Machowicz R, Janka G, Wiktor-Jedrzejczak W. Similar but not the same: differential diagnosis of HLH and sepsis. Crit Rev Oncol Hematol. 2017;114:1-12.

47. Henter JI, Horne A, Arico M, Egeler RM, Filipovich AH, Imashuku S, et al. HLH-2004: diagnostic and therapeutic guidelines for hemophagocytic lymphohistiocytosis. Pediatr Blood Cancer. 2007;48:124-31.

48. Mehta P, McAuley DF, Brown M, Sanchez E, Tattersall RS, Manson JJ, et al. COVID-19: consider cytokine storm syndromes and immunosuppression. Lancet. 2020;395:1033-4.

49. McGonagle D, Sharif K, O'Regan A, Bridgewood C. The role of cytokines including interleukin-6 in COVID-19 induced pneumonia and macrophage activation syndrome-like disease. Autoimmun Rev. 2020;102537. https://doi.org/10.1016/j.autrev.2020. 102537.

50. Zheng M, Gao Y, Wang G, Song G, Liu S, Sun D, et al. Functional exhaustion of antiviral lymphocytes in COVID-19 patients. Cell Mol Immunol. 2020;17:533-5.

51. Zhang W, Zhao Y, Zhang F, Wang Q, Li T, Liu Z, et al. The use of anti-inflammatory drugs in the treatment of people with severe coronavirus disease 2019 (COVID-19): the perspectives of clinical immunologists from China. Clin Immunol. 2020;214:108393.

52. Huang C, Wang Y, Li X, Ren L, Zhao J, Hu Y, et al. Clinical features of patients infected with 2019 novel coronavirus in Wuhan, China. Lancet. 2020;395:497-506.

53. Teijaro JR, Walsh KB, Cahalan S, Fremgen DM, Roberts E, Scott F, et al. Endothelial cells are central orchestrators of cytokine amplification during influenza virus infection. Cell. 2011;146:980-91.

54. Pedersen SF, Ho YC. SARS-CoV-2: a storm is raging. J Clin Investig. 2020;130:2202-5.
55. Wu C, Chen X, Cai Y, Xia J, Zhou X, Xu S, et al. Risk factors associated with acute respiratory distress syndrome and death in patients with coronavirus disease 2019 pneumonia in Wuhan, China. JAMA Intern Med. 2020. https://doi.org/10.1001/jama internmed.2020.0994.

56. Ruan Q, Yang K, Wang W, Jiang L, Song J. Clinical predictors of mortality due to COVID-19 based on an analysis of data of 150 patients from Wuhan, China. Intensive Care Med. 2020. https:// doi.org/10.1007/s00134-020-05991-x.

57. Fletcher AL, Acton SE, Knoblich K. Lymph node fibroblastic reticular cells in health and disease. Nat Rev Immunol. 2015;15:350-61.

58. Koton S, Schneider AL, Rosamond WD, Shahar E, Sang Y, Gottesman RF, et al. Stroke incidence and mortality trends in US communities, 1987 to 2011. JAMA. 2014;312:259-68.

59. Warlow CP. Epidemiology of stroke. Lancet. 1998;352:SIII1-4.

60. Hill MD. Stroke and diabetes mellitus. Handb Clin Neurol. 2014;126:167-74

61. Ungvari Z, Tarantini S, Kirkpatrick AC, Csiszar A, Prodan CI. Cerebral microhemorrhages: mechanisms, consequences, and prevention. Am J Physiol Heart Circ Physiol. 2017;312: H1128-43.

62. Yaghi S, Ishida K, Torres J, Mac Grory B, Raz E, Humbert K, et al. SARS-CoV-2 and stroke in a New York healthcare system. Stroke. 2020;51:2002-11.

63. van Veluw SJ, Shih AY, Smith EE, Chen C, Schneider JA, Wardlaw JM, et al. Detection, risk factors, and functional consequences of cerebral microinfarcts. Lancet Neurol. 2017;16:730-40.

64. Tenembaum SN. Acute disseminated encephalomyelitis. Handb Clin Neurol. 2013;112:1253-62.

65. Reichard RR, Kashani KB, Boire NA, Constantopoulos E, Guo Y, Lucchinetti CF. Neuropathology of COVID-19: a spectrum of vascular and acute disseminated encephalomyelitis (ADEM)-like pathology. Acta Neuropathol. 2020;140:1-6.

66. Karapanayiotides T, Geka E, Prassopoulos P, Koutroulou I, Kollaras P, Kiourtzieva E, et al. Concentric demyelination pattern in COVID-19-associated acute haemorrhagic leukoencephalitis: a lurking catastrophe? Brain. 2020. https://doi.org/10.1093/bra in/awaa375.

67. Handa R, Nanda S, Prasad A, Anand R, Zutshi D, Dass SK, et al. Covid-19-associated acute haemorrhagic leukoencephalomyelitis. Neurol Sci. 2020;41:3023-6.

68. Yong MH, Chan YFZ, Liu J, Sanamandra SK, Kheok SW, Lim $\mathrm{KC}$, et al. A rare case of acute hemorrhagic leukoencephalitis in a COVID-19 patient. J Neurol Sci. 2020;416:117035.

69. Delamarre L, Gollion C, Grouteau G, Rousset D, Jimena G, Roustan J, et al. COVID-19-associated acute necrotising encephalopathy successfully treated with steroids and polyvalent immunoglobulin with unusual IgG targeting the cerebral fibre network. J Neurol Neurosurg Psychiatry. 2020;91:1004-6. 